\title{
Effect of Polymer Charge on Permeability of Urinary Bladder Wall
}

\author{
M. Kerec Kos, M. BogataJ, A. Mrhar \\ Faculty of Pharmacy, University of Ljubljana, Aškerčeva 7, 1000 Ljubljana, Slovenia \\ E-mail: kerecm@ffa.uni-lj.si (M. Kerec Kos) \\ Sci Pharm. 2010; 78: 618 \\ doi:10.3797/scipharm.cespt.8.PDD29
}

The scope of the present work was to establish the role of the polymer charge on its ability to enhance the permeability of the urinary bladder wall.

Cationic poly-L-arginine and chitosan, anionic sodium carboxymethyl cellulose (NaCMC) and alginate as well as nonionic hydroxypropyl cellulose (HPC) and hydroxypropyl methylcellulose (HPMC) were tested. The polymers were tested in concentrations from 0.00005 to $1 \%(\mathrm{w} / \mathrm{v})$ and a concentration of a model drug pipemidic acid (PPA) was $0.014 \%(\mathrm{w} / \mathrm{v})$ in all experiments. All solutions were prepared in phosphate buffer and their $\mathrm{pH}$ was adjusted to 4.5. In diffusion cells the luminal side of isolated pig urinary bladder wall was exposed to tested solutions. In some experiments the tissue was exposed for 60 min to a polymer dispersion with PPA, while in other experiments the tissue was first exposed to a polymer dispersion for $45 \mathrm{~min}$, rinsed and then the solution of PPA was applied for additional $45 \mathrm{~min}$. At the end of the experiment the tissue was frozen and sectioned parallel to luminal surface. PPA was extracted from the tissue segments and its concentration was determined by HPLC.

We showed that the charge of the polymer affects its ability to enhance permeation of PPA into the urinary bladder wall, but to a limited extent. Positively charged chitosan and poly-L-arginine were established as the most promising absorption enhancer into the bladder wall. Compared to chitosan, higher concentrations of poly-L-arginine were needed to significantly increase the tissue permeability. Moreover, poly-L-arginine increased the permeability within 90 min constantly, while chitosan reached the plateau of its absorption enhancement activity after $60 \mathrm{~min}$. In our previous study anionic polymer polycarbophil significantly increased permeation of PPA into the bladder wall [1]. However, in this study anionic NaCMC and alginate did not significantly influence the permeation of PPA into the tissue. Interactions between NaCMC or alginate and PPA might prevail over the potential effect of $\mathrm{NaCMC}$ and alginate on the tissue permeability. Moreover, nonionic polymers HPMC and HPC did not significantly influence the bladder wall permeability.

We can conclude that positive charge has an important influence, but the selection of absorption enhancers for intravesical drug delivery systems can not be done exclusively on the basis of a polymer charge.

[1] Kerec M, Svigelj V, Bogataj M, Mrhar A. The enhancement of pipemidic acid permeation into the pig urinary bladder wall. Int J Pharm. 2002; 240: 33-36. doi:10.1016/S0378-5173(02)00108-4 